\title{
Excluding pulmonary embolism in primary care using the Wells-rule in combination with a point-of care D-dimer test: a scenario analysis
}

\author{
Wim AM Lucassen ${ }^{1 *}$, Renée A Douma ${ }^{2}$, Diane B Toll ${ }^{1}$, Harry R Büller ${ }^{2}$, Henk CPM van Weert $^{1}$
}

\begin{abstract}
Background: In secondary care the Wells clinical decision rule (CDR) combined with a quantitative D-dimer test can exclude pulmonary embolism (PE) safely. The introduction of point-of-care (POC) D-dimer tests facilitates a similar diagnostic strategy in primary care.

We estimated failure-rate and efficiency of a diagnostic strategy using the Wells-CDR combined with a POCD-dimer test for excluding PE in primary care.

We considered ruling out PE safe if the failure rate was $<2 \%$ with a maximum upper confidence limit of $2.7 \%$.

Methods: We performed a scenario-analysis on data of 2701 outpatients suspected of PE. We used test characteristics of two qualitative POC-D-dimer tests, as derived from a meta-analysis and combined these with the Wells-CDR-score.

Results: In scenario 1 (SimpliRed-D-dimer sensitivity 85\%, specificity 74\%) PE was excluded safely in 23.8\% of patients but only by lowering the cut-off value of the Wells rule to <2. (failure rate: 1.4\%, 95\% Cl 0.6-2.6\%) In scenario 2 (Simplify-D-dimer sensitivity $87 \%$, specificity 62\%) PE was excluded safely in $12.4 \%$ of patients provided that the Wells-cut-off value was set at 0. (failure rate: $0.9 \%, 95 \% \mathrm{Cl} 0.2-2.6 \%$ )

Conclusion: Theoretically a diagnostic strategy using the Wells-CDR combined with a qualitative POC-D-dimer test can be used safely to exclude PE in primary care albeit with only moderate efficiency.
\end{abstract}

\section{Background}

Pulmonary embolism (PE) has an estimated annual incidence of 23 cases per 100.000 persons [1]. Because PE is potentially life-threatening, immediate diagnosis and management is essential. As primary care physicians lack accurate diagnostic tools, all patients have to be referred, often with all due speed to secondary care in case PE is suspected. However in $75-95 \%$ of these referred patients PE subsequently is excluded [2-4]. Several management-studies in secondary care have demonstrated that PE can be excluded safely in patients with a low $(<2)$ or unlikely $(\leq 4)$ clinical probability according to the clinical decision rule (CDR) as developed by Wells et al.(Table 1), combined with a normal D-dimer test result (both quantitative and qualitative D-dimer

\footnotetext{
* Correspondence: w.a.lucassen@amc.uva.nl

'Academic Medical Centre, Department of General Practice, Amsterdam, The Netherlands

Full list of author information is available at the end of the article
}

tests) [5-8]. The introduction of easy-to-use rapid pointof-care (POC) D-dimer tests makes it possible to exclude PE safely in the primary care setting, using a diagnostic work-up similar to that in secondary care thereby avoiding unnecessary referrals.

Qualitative POC D-dimer tests do not need additional equipment or calibration, are ready to use, cheap, utilize capillary or venous blood and can be done in-and outside the clinic. They can be interpreted within $10 \mathrm{~min}$ utes as either positive or negative which make the tests suitable for use in primary care. Questions have been raised however about the sensitivity of the tests ranging from $80-100 \%$ in different studies [7,9-13].

To our knowledge a management-study with a diagnostic strategy using a CDR in combination with POCD-dimer test for excluding PE has not been performed in primary care although this approach was successfully used in the setting of suspected deep vein thrombosis (DVT) [14]. We performed a scenario-analysis to 
Table 1 Wells clinical decision rule

\begin{tabular}{ll}
\hline Variable & Points \\
\hline Clinical signs and symptoms of DVT & 3.0 \\
(minimum of leg swelling and pain with palpation of the deep & \\
veins) & \\
\hline Alternative diagnosis less likely than PE & 3.0 \\
\hline Heart rate $>100 /$ min & 1.5 \\
\hline Immobilization (>3 days) or surgery in the previous 4 weeks & 1.5 \\
\hline Previous PE or DVT & 1.5 \\
\hline Haemoptysis & 1.0 \\
\hline $\begin{array}{l}\text { Malignancy } \\
\text { (receiving treatment, treated in the last 6 months or palliative) }\end{array}$ & 1.0 \\
\hline Clinical probability of PE: & \\
Unlikely $\leq 4$ points & \\
Likely $>4$ points \\
Low <2 points \\
Intermediate 2-6 points \\
High >6 points \\
Abbreviations: DVT, deep vein thrombosis; PE, pulmonary embolism
\end{tabular}

calculate the expected results of such a management strategy in patients referred by their primary care physician for suspected PE. Because exclusion of PE is based on the probability score of the Wells rule combined with the result of a qualitative D-dimer test we aimed to calculate a safety-threshold by varying the cut-off value of the Wells-rule.

\section{Methods}

For the present analysis we used data from a large prospective management study, the Christopher-study, including 3306 consecutive in-and outpatients, suspected of pulmonary embolism [8]. This study was performed in secondary care in the Netherlands between November 2002 and September 2004. It evaluated the safety of excluding PE by a sequential diagnostic workup consisting of the dichotomous Wells CDR (cut-off $\leq$ 4), a quantitative D-dimer test and helical computer tomography $(\mathrm{CT})$. Patients with a CDR indicating PE unlikely underwent D-dimer testing. Either the Vidas ELISA D-dimer test or the Tinaquant D-dimer test was used (cut-off $\leq 500 \mu \mathrm{g} / \mathrm{l}$, combined sensitivity $97.8 \%$ and specificity $56.9 \%$ ) and when normal, the diagnosis of $\mathrm{PE}$ was considered excluded. All other patients underwent helical CT. All patients were followed up for a period of 3 months to document the occurrence of subsequent symptomatic venous thrombo-embolism (VTE).

We used test characteristics of two qualitative POC Ddimer tests from a meta-analysis on the diagnostic accuracy of POC-D-dimer tests for excluding VTE [15].

1. SimpliRed D-dimer (sensitivity $85 \%$, specificity $74 \%$ ) is a semi qualitative test performed by mixing capillary or venous blood with a drop of test reagent in the test well. A positive result is defined as any visible agglutination within two minutes.

2. Simplify D-dimer (sensitivity $87 \%$, specificity $62 \%$ ) is a qualitative test and is performed by mixing $35 \mu \mathrm{l}$ of capillary or venous blood with two drops of test reagent. A positive result is indicated by a visible pink-purple coloured line that forms at the test zone. The test can be read within 10 minutes.

To mimic a primary care setting we excluded all inpatients from the original cohort for the present analysis. As would be the case in primary care all patients with Wells CDR $>4$ needed imaging regardless of the D-dimer test result. Hence in these patients no additional D-dimer testing was performed.

Using the original Christopher-study data, we divided the remaining patients into groups according to their individual Wells-CDR scores with different cut-off values (Table 2). Within each group PE was excluded in patients with the combination of a Wells-CDR below the cut-off value and a negative D-dimer test result. Combining the prevalence of $\mathrm{PE}$ in each group with the sensitivity and specificity of the D-dimer test we calculated the theoretical failure-rate and the efficiency of the combined strategy in each clinical probability group.

Efficiency was defined as the proportion of all study patients, in whom PE was excluded (and thus would not need referral) based on a Wells-CDR below various cutoff values and a negative $\mathrm{D}$-dimer test.

The failure rate was defined as the proportion of patients in whom PE was excluded based on a WellsCDR below various cut-off values and a negative D-dimer test, with symptomatic and proven VTE during 3 months follow-up.

We considered ruling out PE safe if the failure rate was $<2 \%$ with a maximum upper confidence limit of $2.7 \%$, being the upper confidence limit of the threemonth thrombo-embolic rate of patients suspected of PE but with a normal pulmonary angiography [16].

The $95 \%$ confidence intervals (CI) were calculated using Confidence Interval Analysis (CIA, version 1.0; Gardner MJ).

\section{Results}

Of the total study population of 3306 in-and outpatients, 2701 were outpatients and included in this analysis. The prevalence of PE in the group of outpatients was $20.2 \%$. (including the 3 -months follow-up period). The prevalence of PE among patients with an unlikely clinical probability decreased with a decreasing CDRcut-off value, ranging from $12.0 \%$ in patients with a Wells score $\leq 4$ to $4.3 \%$ in patients with a Wells score of 0 . 
Table 2 Results of failure-rate and efficiency in $\mathbf{2}$ scenarios at different cut-off values of the Wells-rule in comparison with results of the Christopher-study

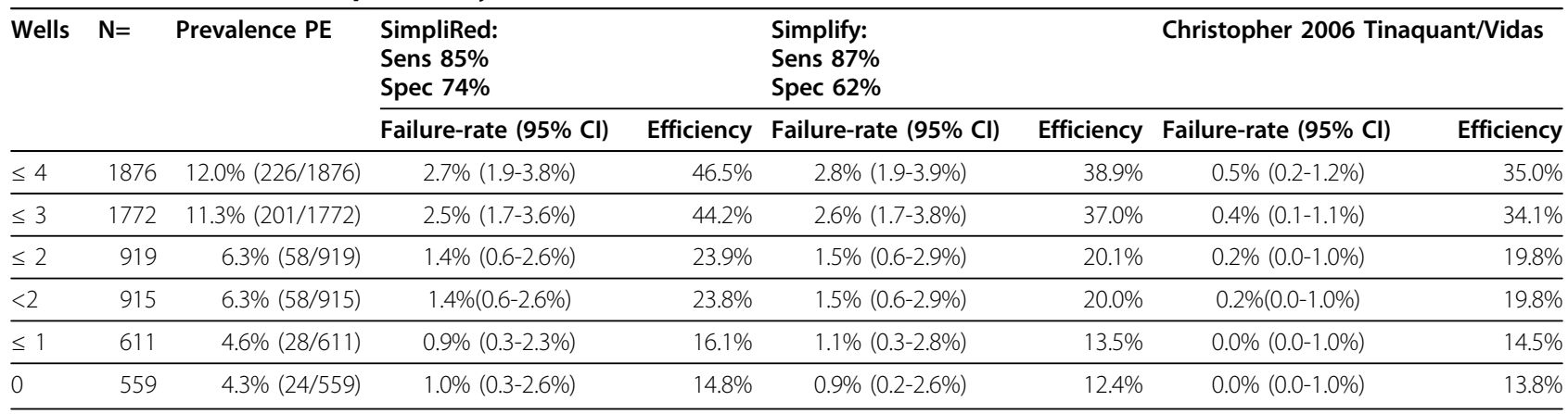

$\mathrm{N}=$ Number of outpatients in different Wells clinical probability groups

$\mathrm{Cl}=$ Confidence interval

Table 2 shows the failure-rate and the efficiency at different cut-off values of the Wells-CDR in combination with the sensitivity and specificity of the D-dimer test. In the last column results of the outpatients obtained from the Christopher-study are depicted for comparison. In the Christopher-study PE could be excluded safely with a Wells-CDR cut-off value of $\leq 4$ in $35.0 \%$ of the patients.

However the failure rate of $2.7 \%$ is exceeded in both qualitative POC D-dimer tests when combined with a Wells-CDR cut off value of $\leq 4$. To meet the safety criteria (failure rate $<2 \%$, upper $95 \% \mathrm{CI}<2.7 \%$ ) the SimpliRed D-dimer test had to be combined with a Wells CDR-cut off value $<2$ and the Simplify D-dimer test with a Wells CDR-cut off value of 0 . Using this strategy, the proportion of patients in whom PE might be excluded safely decreased to $23.8 \%$ with the SimpliRed D-dimer test and $12.4 \%$ with the Simplify D-dimer test. The dramatic loss in efficiency when using a lower Wells-CDR cut off is demonstrated in figure 1.

\section{Discussion}

The current scenario-analysis determined the theoretical failure-rate and efficiency of a diagnostic strategy using the Wells CDR at different cut-off values combined with a qualitative POC D-dimer test for excluding PE in primary care. Excluding PE safely in primary care with a CDR and a point-of-care D-dimer test seems feasible. However, the strategy appeared to be safe only when the cut-off value of the Wells-CDR was lowered to $<2$ using the SimpliRed and 0 using the Simplify D-dimer test, respectively. Efficiency is considerably lower when using those cut-off values: the number of patients that need referral is $76.2 \%$ and $87.6 \%$ respectively, as compared to $65 \%$ with the Wells cut-off value of $\leq 4$ in the Christopher-study.

Several aspects of this analysis require comment.
Firstly, we based the analysis on the test characteristics of two qualitative POC-D-dimer tests as reported in a diagnostic meta-analysis. In this meta-analysis most of the studies included patients suspected of DVT. Only six studies included patients with PE. However in a covariate analysis of studies with only DVT both the sensitivity and the specificity of the SimpliRed and the Simplify D-dimer test were essentially the same as in the overall analysis.

Secondly, several studies performed in secondary care (PE-prevalence ranging from $3.8-10 \%$ ) show that a strategy using a CDR and a qualitative POC-D-dimer test can be used safely to exclude PE. Moreover these studies show a good efficiency ranging from 44-66\% [7,10-13]. Wells et al were the first to show that the combination of Wells CDR $<2$ and a negative D-dimer test was safe to exclude PE. (prevalence 9.5\%, failure rate $0.2 \%$, efficiency 47\%) [7]. According to Hogg and co-workers the Simplify D-dimer test alone was not sufficiently sensitive (sensitivity $81.8 \%$, specificity $74.2 \%$ ) to exclude PE in low-risk patients (prevalence PE 5.3\%) presenting to the emergency department (ED) with pleuritic chest pain. However, when the Simplify D-dimer test was combined with a low-clinical probability Wells-rule the negative predictive value of the combined test was 99.3\% (CI 97.499.9\%): high enough to exclude PE safely [10]. Kline et al showed in low-risk ED-patients (prevalence PE 4.7\%) that combination of a physician's unstructured estimate of pre-test probability of PE of $<15 \%$ and a negative Simplify-D-dimer test excluded PE safely (sensitivity D-Dimer-test $80.6 \%$, specificity $72.5 \%$ ) [11]. In a primary care based management study sensitivity of the Simplify D-dimer test proved to be sufficient to exclude deep vein thrombosis (DVT) safely in patients with a low clinical probability. The relatively higher specificity, as compared to laboratory based quantitative D-dimer tests provided a good efficiency [14]. 


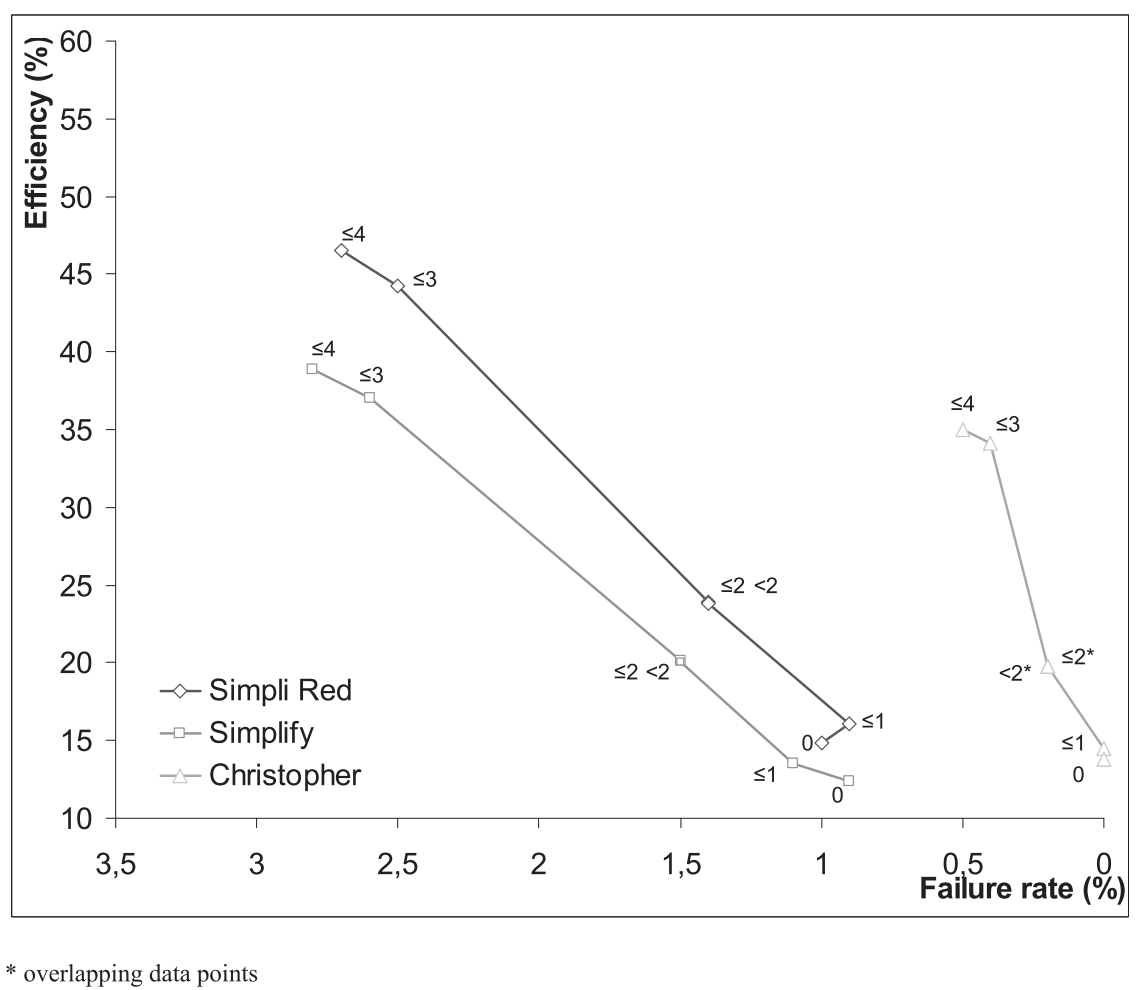

Figure 1 Failure rate versus efficiency in 2 scenarios at different cut-off values of the Wells CDR in comparison with results from the Christopher-study.

Although the sensitivity of the Simplify D-Dimer test in the studies of Hogg and Kline was only $81.8 \%$ and $80.6 \%$, respectively, the negative predictive value of the combined strategy using a pre-test probability assessment and the Simplify D-Dimer test was high enough to exclude PE safely due to the low PE-prevalence in these studies.

Thirdly, a weak point of the analysis is that although we have excluded all in-patients the study-population is still not really a primary care population. The outpatients included in the Christopher-study are likely selectively biased as the primary care physician used his own judgement before referring the patient. In the Christopher-study the PE-prevalence was $20.2 \%$. In daily practice when a primary care physician will use the Wells-CDR rule combined with a POC D-dimer test the prevalence of PE in suspected patients is expected to be lower which will improve the negative predictive value (and thereby safety and efficiency) of an exclusion strategy for PE in primary care.

Fourthly, we don't know how well the Wells CDR would perform in primary care. In secondary care the Wells rule is usually applied after routine blood tests, chest radiography and electrocardiography. The primary care physician is usually lacking this information and this will clearly influence the scoring of the subjective variable 'pulmonary embolism is as likely as or more likely than an alternative diagnosis'.
Fifthly, we know that the test characteristics of the POC-D-dimer test, unlike this scenario, are not fixed but are influenced by the prevalence of PE in the different Wells-groups. It is likely that the specificity of the D-dimer test will increase as the prevalence decreases. This might improve the negative predictive value of the strategy in primary care $[17,18]$.

Sixthly, in this analysis the SimpliRED D-dimer assay was used which has certain limitations. It is known that this method may be associated with a risk for inadequate interpretation due to the fact that the results are based on a subjective interpretation of the presence or absence of agglutination [19]. This risk for inadequate interpretation will be enhanced by infrequent use of the assay. An average Dutch primary care physician will use a POC D-dimer assay for exclusion of PE only 3-5 times a year. However the physician will use the same assay also for exclusion of DVT [14]. We expect the Dutch primary care physician to apply the POC D-dimer test 12-15 times a year in both suspected PE-patients as DVT-patients. We think this will justify an adequate and reliable use of the assay.

Finally, although in scenario 2 (Simplify) the point estimate failure rate in Wells CDR $<2$ is within the safety limits, the upper confidence limit exceeds $2.7 \%$. Confidence intervals become larger with decreasing 
number of patients. It can be expected that with an increasing number of patients the proportion in the lower Wells-CDR score will be higher and the confidence interval will become narrower. Therefore scenario 2 might also be safe in Wells $<2$.

In secondary care, in a strategy using a more sensitive, quantitative D-dimer test, a cut-off value of Wells $\leq 4$ is generally accepted as safe. Although the sensitivity of the POC qualitative D-dimer test is lower, the specificity of the test is higher and as a consequence efficiency is higher at the cost of safety. Recalibration of the Wellsrule for a primary care situation might overcome the safety problems.

\section{Conclusion}

In this scenario-analysis we could exclude PE safely with a diagnostic strategy using the Wells CDR and a qualitative D-dimer test, albeit with only a moderate efficiency. A prospective study is needed to assess safety and efficiency of this strategy in a true primary care population. Recalibration of the Wells-rule or adaption of cut-off values might then be needed.

\section{Acknowledgements}

Funding: Financial support was provided by the Netherlands Heart Foundation (project number No.2006B237). This had no influence on any aspect of the study.

\section{Author details}

${ }^{1}$ Academic Medical Centre, Department of General Practice, Amsterdam, The Netherlands. ${ }^{2}$ Academic Medical Centre, Department of Vascular Medicine, Amsterdam, The Netherlands.

\section{Authors' contributions}

$\mathrm{RD}, \mathrm{WL}$ had full access to all of the data in the study and take responsibility for the integrity of the data and the accuracy of the data analysis. Study concept and design: HvW, WL. Acquisition of data: RD, WL. Analysis and interpretation of data: DT, HvW, WL. Drafting of the manuscript: HvW, WL. Critical revision of the manuscript for important intellectual content: DT, HB, HvW, RD, WL. Statistical analysis: WL. Obtaining funding: HvW. Study supervision: HvW, WL. All authors read and approved the final manuscript. Data used in this document is openly available.

\section{Competing interests}

The authors declare that they have no competing interests.

Received: 10 May 2010 Accepted: 13 September 2010 Published: 13 September 2010

\section{References}

1. Anderson FA Jr, Wheeler HB, Goldberg RJ, Hosmer DW, Patwardhan NA, Jovanovic B, et al: A population-based perspective of the hospital incidence and case-fatality rates of deep vein thrombosis and pulmonary embolism. The Worcester DVT Study. Arch Intern Med 1991, 151:933-938.

2. Runyon MS, Webb WB, Jones $A E$, Kline JA: Comparison of the unstructured clinician estimate of pretest probability for pulmonary embolism to the Canadian score and the Charlotte rule: a prospective observational study. Acad Emerg Med 2005, 12:587-593.

3. Perrier A, Roy PM, Aujesky D, Chagnon I, Howarth N, Gourdier AL, et al: Diagnosing pulmonary embolism in outpatients with clinical assessment, D-dimer measurement, venous ultrasound, and helical computed tomography: a multicenter management study. Am J Med 2004, 116:291-299.

4. Penaloza A, Melot C, Dochy E, Blocklet D, Gevenois PA, Wautrecht JC, et al: Assessment of pretest probability of pulmonary embolism in the emergency department by physicians in training using the Wells model. Thromb Res 2007, 120:173-179.

5. Anderson DR, Kovacs MJ, Dennie C, Kovacs G, Stiell I, Dreyer J, et al: Use of spiral computed tomography contrast angiography and ultrasonography to exclude the diagnosis of pulmonary embolism in the emergency department. J Emerg Med 2005, 29:399-404.

6. Goekoop RJ, Steeghs N, Niessen RW, Jonkers GJ, Dik H, Castel A, et al: Simple and safe exclusion of pulmonary embolism in outpatients using quantitative D-dimer and Wells' simplified decision rule. Thromb Haemost 2007, 97:146-150.

7. Wells PS, Anderson DR, Rodger M, Stiell I, Dreyer JF, Barnes D, et al: Excluding pulmonary embolism at the bedside without diagnostic imaging: management of patients with suspected pulmonary embolism presenting to the emergency department by using a simple clinical model and d-dimer. Ann Intern Med 2001, 135:98-107.

8. Belle van A, Buller HR, Huisman MV, Huisman PM, Kaasjager K, Kamphuisen PW, et al: Effectiveness of managing suspected pulmonary embolism using an algorithm combining clinical probability, D-dimer testing, and computed tomography. JAMA 2006, 295:172-179.

9. Kline JA, Israel EG, Michelson EA, O'Neil BJ, Plewa MC, Portelli DC: Diagnostic accuracy of a bedside D-dimer assay and alveolar dead-space measurement for rapid exclusion of pulmonary embolism: a multicenter study. JAMA 2001, 285:761-768.

10. Hogg K, Dawson D, kway-Jones $K$ : The emergency department utility of Simplify D-dimer to exclude pulmonary embolism in patients with pleuritic chest pain. Ann Emerg Med 2005, 46:305-310.

11. Kline JA, Runyon MS, Webb WB, Jones AE, Mitchell AM: Prospective study of the diagnostic accuracy of the simplify D-dimer assay for pulmonary embolism in emergency department patients. Chest 2006, 129:1417-1423.

12. Toulon $P$, Lecourvoisier $C$, Meyniard $O$ : Evaluation of a rapid qualitative immuno-chromatography D-dimer assay (Simplify D-dimer) for the exclusion of pulmonary embolism in symptomatic outpatients with a low and intermediate pretest probability. Comparison with two automated quantitative assays. Thromb Res 2009, 123:543-549.

13. Runyon MS, Beam DM, King MC, Lipford EH, Kline JA: Comparison of the Simplify D-dimer assay performed at the bedside with a laboratorybased quantitative D-dimer assay for the diagnosis of pulmonary embolism in a low prevalence emergency department population. Emerg Med J 2008, 25:70-75.

14. Buller HR, Ten Cate-Hoek AJ, Hoes AW, Joore MA, Moons KG, Oudega R, et al: Safely ruling out deep venous thrombosis in primary care. Ann Intern Med 2009, 150:229-235.

15. Geersing GJ, Janssen KJ, Oudega R, Bax L, Hoes AW, Reitsma JB, et al: Excluding venous thromboembolism using point of care D-dimer tests in outpatients: a diagnostic meta-analysis. BMJ 2009, 339:b2990.

16. van Beek EJ, Brouwerst EM, Song B, Stein PD, Oudkerk M: Clinical validity of a normal pulmonary angiogram in patients with suspected pulmonary embolism-a critical review. Clin Radiol 2001, 56:838-842.

17. Fletcher Robert H, Fletcher Suzanne W: Clinical epidemiology, the essentials 4th edition, 42005.

18. Wolf SJ, McCubbin TR, Nordenholz KE, Naviaux NW, Haukoos JS: Assessment of the pulmonary embolism rule-out criteria rule for evaluation of suspected pulmonary embolism in the emergency department. Am J Emerg Med 2008, 26:181-185.

19. Perzanowski $C$, Eiger G: Limited interobserver agreement in the SimpliRED D-dimer assay. J Thromb Haemost 2003, 1:849-850.

Pre-publication history

The pre-publication history for this paper can be accessed here: http://www.biomedcentral.com/1471-2296/11/64/prepub

doi:10.1186/1471-2296-11-64

Cite this article as: Lucassen et al:: Excluding pulmonary embolism in primary care using the Wells-rule in combination with a point-of care D-dimer test: a scenario analysis. BMC Family Practice 2010 11:64. 